\title{
Hyperbolic Systems of Equations Posed on Erroneous Curved Domains
}

\author{
Jan Nordström ${ }^{\mathrm{a}}$, Samira Nikkar ${ }^{\mathrm{b}}$ \\ ${ }^{a}$ Department of Mathematics, Computational Mathematics, Linköping University, SE-581 83 \\ Linköping, Sweden ( jan.nordstrom@liu.se). \\ ${ }^{b}$ Department of Mathematics, Computational Mathematics, Linköping University, SE-581 83 \\ Linköping, Sweden (samira.nikkar@liu.se).
}

\begin{abstract}
The effect of an inaccurate geometry description on the solution accuracy of a hyperbolic problem is discussed. The inaccurate geometry can for example come from an imperfect CAD system, a faulty mesh generator, bad measurements or simply a misconception.

We show that inaccurate geometry descriptions might lead to the wrong wave speeds, a misplacement of the boundary conditions, to the wrong boundary operator and a mismatch of boundary data.

The errors caused by an inaccurate geometry description may affect the solution more than the accuracy of the specific discretization techniques used. In extreme cases, the order of accuracy goes to zero. Numerical experiments corroborate the theoretical results.
\end{abstract}

\section{Erroneous computational domain}

Consider the following hyperbolic system of equations, in two space dimensions,

$$
\begin{aligned}
W_{t}+\hat{A} W_{x}+\hat{B} W_{y} & =0, & & (x, y) \in \Omega, & & t \in(0, T], \\
L W & =g(x, y, t), & & (x, y) \in \delta \Omega, & & t \in(0, T], \\
W & =f(x, y), & & (x, y) \in \Omega, & & t=0,
\end{aligned}
$$

in which the solution is represented by the vector $W=W(x, y, t) . \hat{A}$ and $\hat{B}$ are constant symmetric $M \times M$ matrices, $\Omega$ is the spatial domain with the boundary $\delta \Omega$. The boundary operator $L$ is defined on $\delta \Omega, f(x, y) \in \mathbb{R}^{M}$ and $g(x, y, t) \in \mathbb{R}^{M}$ are the data in the problem. 
Equation (1) is transformed to curvilinear coordinates $(\xi, \eta)$ by $\left(V_{\xi}, V_{\eta}, V_{t}\right)=$ $[J]\left(V_{x}, V_{y}, V_{t}\right)^{T}$, where $[J]$ is the Jacobian matrix of the transformation. The transformed problem is

$$
\begin{aligned}
& J W_{t}+A W_{\xi}+B W_{\eta}=0, \quad(\xi, \eta) \in \Phi, \quad t \in(0, T], \\
& L W=g(\xi, \eta, t), \quad(\xi, \eta) \in \delta \Phi, \quad t \in(0, T], \\
& W=f(\xi, \eta), \quad(\xi, \eta) \in \Phi, \quad t=0,
\end{aligned}
$$

where $A=J \xi_{x} \hat{A}+J \xi_{y} \hat{B}, B=J \eta_{x} \hat{A}+J \eta_{y} \hat{B}$ and $J=x_{\xi} y_{\eta}-x_{\eta} y_{\xi}>0$ is the determinant of $[J]$.

The energy method together with the metric identities and the use of the Green-Gauss theorem yields

$$
\frac{d}{d t}\|W(\xi, \eta, t)\|_{J}^{2}=-\oint_{\delta \Phi} W^{T} C W d s,
$$

where the norm is defined by $\|V\|_{J}^{2}=\iint_{\Phi} V^{T} J V d \xi d \eta$. In (3), $C=(A, B) \cdot n=$ $\alpha A+\beta B=X \Lambda X^{T}, n=(\alpha, \beta)$ is the unit normal vector pointing outward from $\Phi, X$ contains the eigenvectors as columns and $\Lambda$ contains the eigenvalues on the main diagonal. For more details see [1].

The problem (2) is well-posed if we impose characteristic boundary conditions and the following energy rate is obtained

$$
\frac{d}{d t}\|W(\xi, \eta, t)\|_{J}^{2}+\oint_{\delta \Phi} W^{T} C^{+} W d s=\oint_{\delta \Phi} g^{T}\left|\Lambda^{-}\right| g d s,
$$

where $\Lambda^{-}$contains the negative eigenvalues of $C$ and $C^{+}=X \Lambda^{+} C^{T}$.

\subsection{Errors due to the wrong position of boundary}

Consider two hyperbolic problems with solutions $U$ and $V$ posed on two nearby domains. The two domains, depicted in Figure 1, are both mapped to the unit square. We consider $\Omega_{c}$ to be the correct domain and $\Omega_{e}$ to be the erroneous one (subscripts c and e denote correct and erroneous, respectively). The transformed equations become

$$
J_{c} U_{t}+A_{c} U_{\xi}+B_{c} U_{\eta}=0, J_{e} V_{t}+A_{e} V_{\xi}+B_{e} V_{\eta}=0,
$$

where the matrices are $A_{i}=\left[J \xi_{x} \hat{A}+J \xi_{y} \hat{B}\right]_{i}$ and $B_{i}=\left[J \eta_{x} \hat{A}+J \eta_{y} \hat{B}\right]_{i}$, for $i=c$,e. Our first result follows immediately. Since $A_{c} \neq A_{e}, B_{c} \neq B_{e}$ we realize that:

1) The wave-speeds given by the eigenvalues of the matrices will differ, and that $V$ will have the wrong wave speeds. 


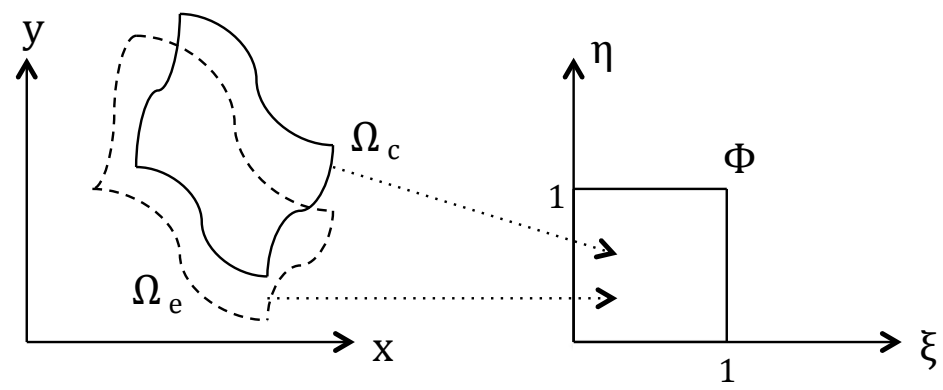

Figure 1: A schematic of two different geometry descriptions both mapped to the unit square.

Next, we consider the results of the energy method given by (4) where $W \in\{U, V\}$ and $C_{i}=\alpha_{i} A_{i}+\beta_{i} B_{i}$ for $i=c, e$. A closer look at the boundary term $C_{i}$ reveals that the normals as well as the eigenvalues of $C_{i}$ are modified by the erroneous geometry. This paves the way for the second, third and fourth conclusions:

2) Error in the normal: This error is caused by imposing the wrong boundary operator due to an erroneous normal. Consider for example the solid wall no penetration condition $(u, v) \cdot n=\alpha u+\beta v=g=0$ for the Euler equations. An erroneous normal will lead to the wrong boundary operator. This means that the boundary operator $L$ in (2) is wrong while the data $g$ is correct. See case 1 in Figure 2.

3) Error in the position: Here the error is due to the misplacement of the boundary condition. Consider again the solid wall no penetration condition for the Euler equations, now with a correct boundary operator and data, imposed at the wrong position in space. In this case, the boundary operator $L$ and the data $g$ in (2) are both correct. See case 2 in Figure 2.

4) Error in the data: Boundary conditions with data from $\Omega_{c}$ but imposed at $\Omega_{e}$ will also lead to inaccurate results. In this case, the data $g$ in (2) is wrong while the boundary operator $L$ can be either correct or wrong. See case 2 and 3 in Figure 2.

We can also have a combination of all the errors. These errors might be, and often are [4], more important than the order of accuracy of the specific discretization techniques used (which will be shown later in the numerical experiments section below).

To further discuss the effect of the wrong position of boundary conditions, consider (2) and two types of boundary data, correct and erroneous denoted by $g_{c}$ and $g_{e}$, respectively. The correct and erroneous solutions are denoted by $U$ and $V$, respectively, and for simplicity we assume that they have the same initial 
(c) $=$ correct geometry

(e) = erroneous geometry

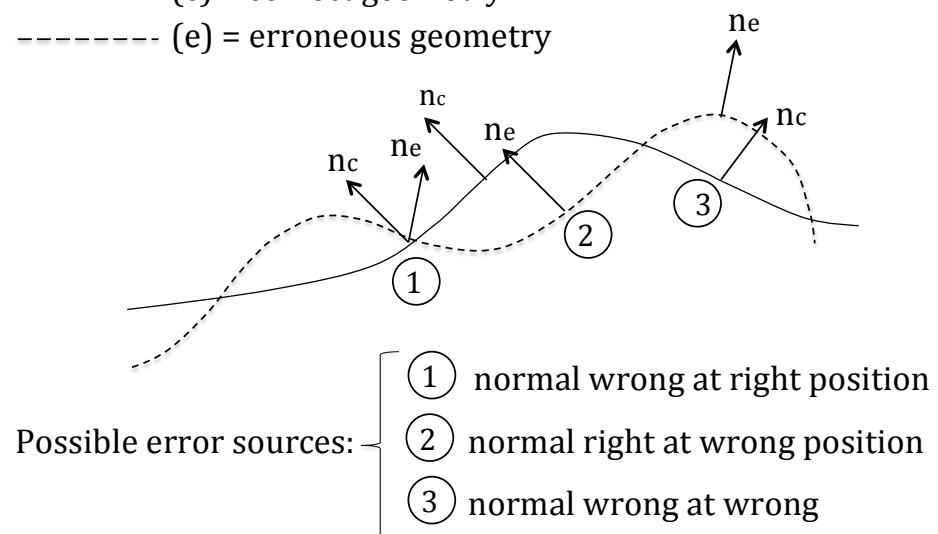

Figure 2: A schematic of the erroneous and correct $\left(n_{c}=\right.$ correct normal, $n_{e}=$ erroneous normal) boundary definitions.

data $f$ which vanishes close to the boundaries. By subtraction we obtain an error equation with zero initial data and non-zero boundary data. Let $E=U-V$ denote the error, and

$$
\delta g=g_{e}-g_{c}=(\nabla g)_{e} \cdot \overrightarrow{d x}+\mathscr{O}\left(|\overrightarrow{d x}|^{2}\right)=\mathscr{O}(|\overrightarrow{d x}|) .
$$

In (6), $\overrightarrow{d x}=(d x, d y), d x=x_{c}-x_{e}, d y=y_{c}-y_{e}$, see Figure 3 .

(c) = correct geometry

(e) = erroneous geometry

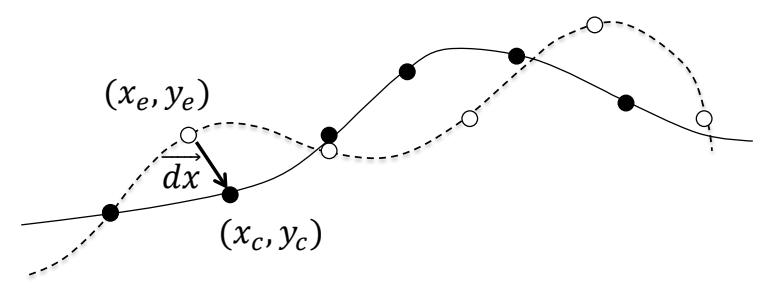

Figure 3: A blow-up of two nearby boundary points which result in two types of data, $g_{c}=$ $g\left(x_{c}, y_{c}, t\right)$ and $g_{e}=g\left(x_{e}, y_{e}, t\right)$.

The energy method applied to the transformed version of the error equation gives the corresponding energy rate to (4), as

$$
\frac{d}{d t}\|E\|_{J_{e}}^{2}+\oint_{\delta \Phi} E^{T} C_{e}^{+} E d s=-\oint_{\delta \Phi} \delta g^{T} \Lambda_{e}^{-} \delta g d s
$$


in which $\Lambda_{e}^{-}$contain the negative eigenvalues of $C_{e}$. From (7) we can conclude that the error is bounded for long time calculations, see [3] for details.

\section{Numerical experiments}

To quantify the error stemming from an erroneous geometry we consider the geometries in Figures 4 and 5. The geometry description is given by

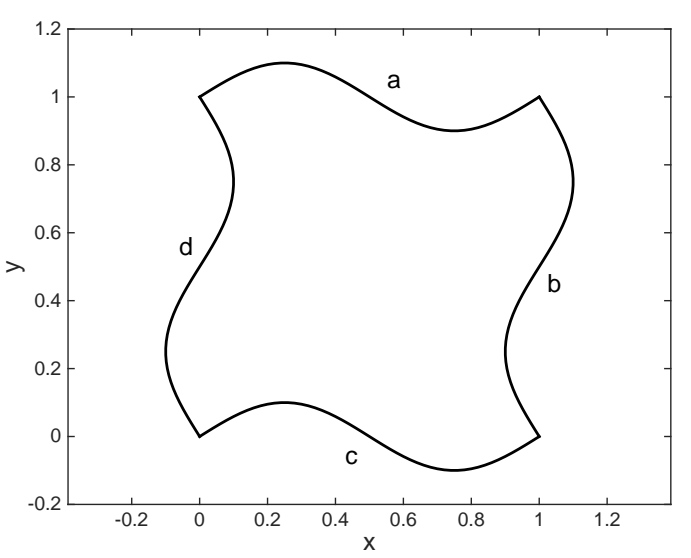

Figure 4: The correct domain, $\Omega_{c}$.

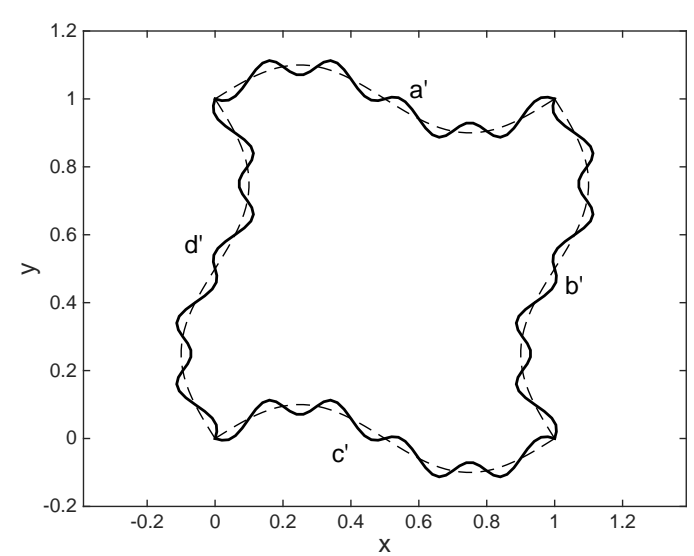

Figure 5: The erroneous domain, $\Omega_{e}$.

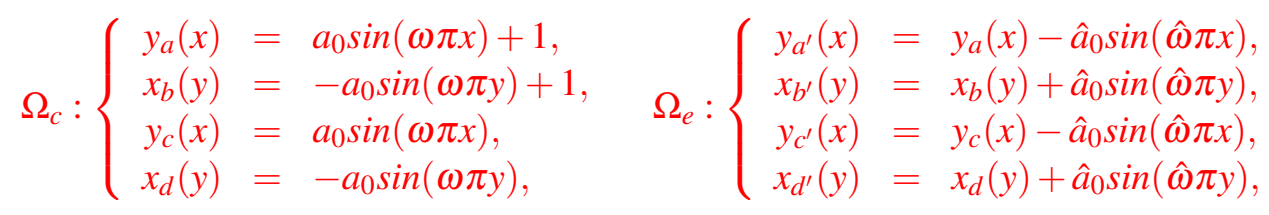

where $a_{0}=0.1, \omega=2$ and $\hat{\omega}=20$. Moreover, $\hat{a}_{0}=0.1$ for $q=0$ and $\hat{a}_{0}=10|\overrightarrow{d x}|^{q}$ for $q \in\{1,2,3\}$. Note that $\Omega_{e}$ in Figure 5 is exaggerated for a better visualization, and $\Omega_{c}$ is included for clarity. It is not used during the numerical experiments. We consider the two-dimensional constant coefficient symmetrized Euler equations in which $V=[\rho, u, v, T]^{T}$ is the perturbed solution. The components $\rho, u, v$, and $T$ are respectively the density, the $\mathrm{x}$ and $\mathrm{y}$ velocity components and the temperature perturbation. The matrices $\hat{A}$ and $\hat{B}$ are given in [1].

To verify the accuracy of our computational method, we use the manufactured solution $V_{\infty}=[\sin (\theta), \cos (\theta), \sin (\phi), \cos (\phi)]^{T}$, where $\theta(x, t)=10 x-5 t$, $\phi(y, t)=5 y-10 t$. The manufactured solution is injected in the problem through a forcing function, boundary and initial data. We use characteristic boundary conditions as in the derivations above. The convergence rates using a finite difference operator on summation-by-parts form (SBP84) in space and time with weak 
boundary and initial conditions, are shown in Table 1. SBP84 in space and time converges with 5th order and the convergence rates are clearly correct [2].

Table 1: Convergence rates for the problem (1) with correct data

\begin{tabular}{lcccccc}
\hline Number of grid points & 31 & 41 & 51 & 61 & 71 & 81 \\
\hline The $\rho$ component & 4.4982 & 4.4087 & 4.5281 & 4.6254 & 4.6963 & 4.7487 \\
The $u$ component & 4.6578 & 4.6186 & 4.6222 & 4.6603 & 4.7141 & 4.7727 \\
The $v$ component & 4.9240 & 5.0875 & 5.0093 & 4.9176 & 4.8635 & 4.8394 \\
The $T$ component & 5.3118 & 5.3419 & 5.1547 & 4.9906 & 4.8840 & 4.8055 \\
\hline
\end{tabular}

Equipped with a 5th order convergent computational scheme (we use SBP84 in space and time, see [1] for details on this technique), we proceed to investigate the problem with erroneous data. We compute the erroneous solution by computing on the erroneous domain $\Omega_{e}$, taking the boundary data from the nearby domain $\Omega_{c}$ and finally subtracting the exact manufactured solution. The convergence rates for the erroneous solution are shown in Figure 6. We have considered different magnitudes for the deviation of the erroneous domain from the correct domain in terms of $|\overrightarrow{d x}|=\mathscr{O}\left(h^{q}\right)$ where $h$ is the grid spacing and $q \in\{0,1,2,3\}$. As seen in Figure 6, imposing the boundary data at an erroneous position affects the results and reduces the global order accuracy of the scheme to $q$. Finally, in Figure 7 we show how the error behaves in long time calculations. It reaches an error bound as predicted above, since we use characteristic boundary conditions as shown in [3].

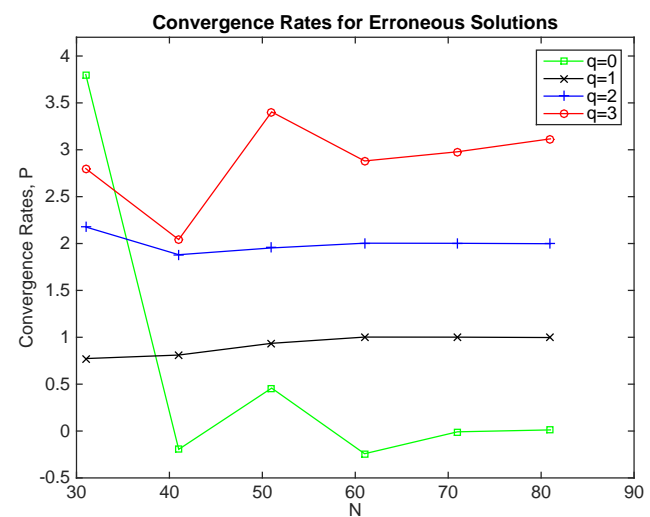

Figure 6: Convergence rates for the erroneous solutions in $\Omega_{e}$ with $q \in\{0,1,2,3\}, \mathrm{T}=1$ and sufficiently small time steps.

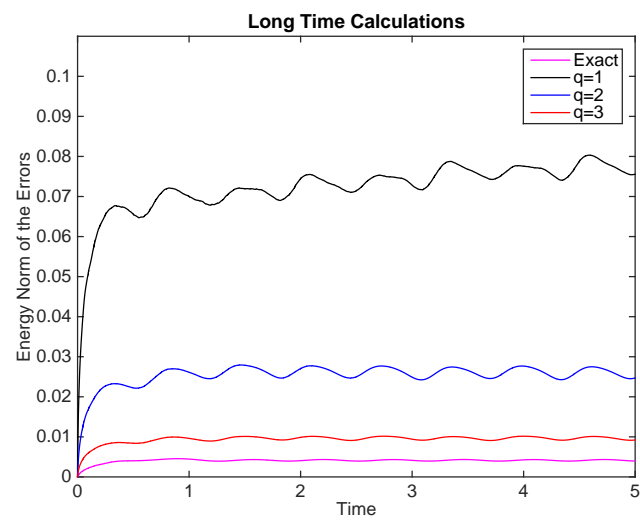

Figure 7: Error versus time for exact and erroneous solutions with $q \in\{1,2,3\}, 31$ and 1501 grid points in space and time, respectively. 


\section{Summary and conclusions}

The effects of an erroneous geometry description were discussed by using the energy method as the analytical tool. We derived an error estimate which describes the effects of imposing correct boundary data at erroneous boundary positions.

We used a 5 th order provable stable and convergent high order finite difference method as our computational tool. The results obtained are valid for all discretization techniques, that are sufficiently high order accurate and stable.

We conclude that an erroneous geometry description might lead to the wrong wave speeds, a misplacement of the boundary conditions, to the wrong boundary operator and a mismatch of boundary data.

The errors caused by an inaccurate geometry description may affect the solution more than the accuracy of the specific discretization techniques used. In extreme cases, the order of accuracy goes to zero. The numerical experiments corroborate this conclusion.

\section{References}

[1] S. Nikkar and J. Nordström, Fully discrete energy stable high order finite difference methods for hyperbolic problems in deforming domains, Journal of Computational Physics, 291(0):82-98 (2015).

[2] M. Svärd and J. Nordström, Review of summation-by-parts schemes for initial-boundary-value problems, Journal of Computational Physics, Volume 268, pp. 1738 (2014).

[3] J. Nordström, Error bounded schemes for time-dependent hyperbolic problems, SIAM Journal of Scinetific Computing, 30:46-59, (2007).

[4] I. Babuska and J. Chleboun, Effects of uncertanities in the domain on the solution of Neumann boundary value problems in two spatial dimensions, Math. Comput., 71:1339-1370, (2002). 